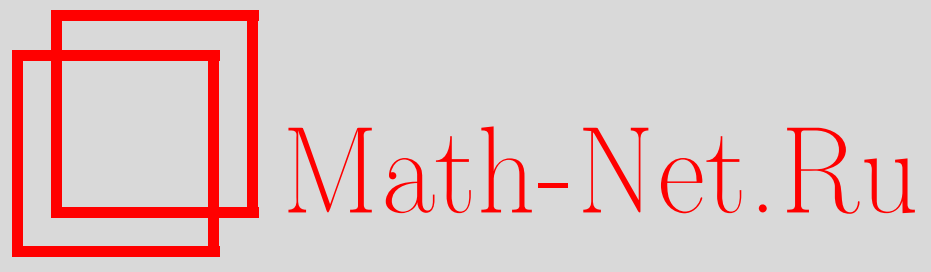

М. М. Драгилев, П. А. Чалов, О пространствах Фреше с безусловным базисом, Матем. заметки, 2006, том 80, выпуск 1, 29-32

DOI: https://doi.org/10.4213/mzm2776

Использование Общероссийского математического портала Math-Net.Ru подразумевает, что вы прочитали и согласны с пользовательским соглашением http://www . mathnet.ru/rus/agreement

Параметры загрузки:

IP : 54.157 .27 .8

26 апреля 2023 г., 14:09:27

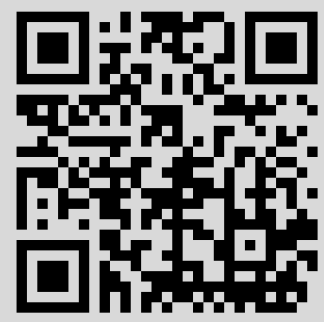




\section{О ПРОСТРАНСТВАХ ФРЕШЕ \\ С БЕЗУСЛОВНЫМ БАЗИСОМ}

\section{М. М. Драгилев, П. А. Чалов}

Ненормируемое $\mathscr{F}$-пространство, имеющее безусловный базис, не обязательно изоморфно обобщенному пространству Кёте.

Библиография: 23 названия.

Пусть $X$ - отделимое локально выпуклое пространство (ЛВП) с определяющей системой преднорм $\left(|x|_{p}: x \in X, p \in \mathscr{P}\right),\left(x_{n}\right)_{1}^{\infty} \subset X-$ последовательность отличных от нуля элементов, $\ell$ - банахово пространство последовательностей $\left(t_{n}\right)_{1}^{\infty}$ комплексных чисел с безусловным базисом ортов $\left(e_{n}\right)_{1}^{\infty}, e_{n}=\left(\delta_{i n}\right)_{i=1}^{\infty}, n=1,2, \ldots$, и с монотонной нормой $\|\cdot\|$, удовлетворяющей условию: $\left\|e_{n}\right\|=1, n=1,2, \ldots$ (сокращенно, $\ell \in \Lambda$ ).

Обобщенным пространством Кёте (ОПК) [1; § 3] называют векторное пространство

$$
\ell\left(\left(a_{p n}\right)_{\mathscr{P}, \mathbb{N}}\right) \quad\left(=\ell\left(\left(x_{n}\right)_{\mathbb{N}}\right)\right) \quad\left(a_{p n}=\left|x_{n}\right|_{p}\right)
$$

всех числовых последовательностей $\left(t_{n}\right)_{1}^{\infty}$ таких, что $\left(t_{n} a_{p n}\right)_{n=1}^{\infty} \in \ell(p \in \mathscr{P})$ с локально выпуклой топологией, задаваемой системой преднорм

$$
\left|\left(t_{n}\right)_{1}^{\infty}\right|_{p}^{*}=\left\|\left(t_{n} a_{p n}\right)_{\mathbb{N}}\right\|, \quad p \in \mathscr{P} .
$$

Последовательность ортов является в этом пространстве безусловным шаудеровским базисом. Если $\left(x_{n}\right)_{1}^{\infty}$ - базис в $X$ и система преднорм

$$
\left(|x|_{p}^{*}=\left|\left(t_{n}\right)_{1}^{\infty}\right|_{p}^{*}: x=\sum_{n} t_{n} x_{n} \in X, \quad p \in \mathscr{P}\right)
$$

задает исходную топологию в $X$, то базис $\left(x_{n}\right)_{1}^{\infty}$ называют $\ell$-абсолютным (или базисом типа $\ell)$. При этом пространство $X$ изоморфно соответствующему ОПК: $X \simeq \ell\left(\left(x_{n}\right)_{\mathbb{N}}\right)$. Например, безусловный базис $\left(x_{n}\right)_{1}^{\infty}$ банахова пространства $X$ является $\ell$-абсолютным базисом, где $\ell \in \Lambda$ определяется соотношениями

$$
\ell=\left\{\left(t_{n}\right)_{1}^{\infty}: \sum_{n} t_{n} \frac{x_{n}}{\left\|x_{n}\right\|}=x \in X\right\}, \quad\left\|\left(t_{n}\right)_{1}^{\infty}\right\|=\|x\| .
$$

Таким образом, каждое (бесконечномерное) банахово пространство с безусловным базисом изоморфно некоторому ОПК.

(C) М. М. ДРАГилЕв, П. А. ЧАЛОв, 2006 
ЗАмЕчаниЕ. 1. Абсолютный шаудеровский базис в бочечном ЛВП имеет тип $\ell_{1}$.

2. Изоморфные ЛВП могут иметь $\ell$-абсолютные базисы не совпадающих типов. Таковы, например, любые два не эквивалентные безусловные нормированные базиса в банаховом пространстве.

3. В ядерном $\mathscr{F}$-пространстве, имеющем базис, все базисы абсолютны [2], а также $\ell$-абсолютны при $\ell \in \Lambda$.

Как вытекает из [3]-[16] (см. также [1], [17]), существуют три и только три базисных типа $-\ell_{1}, \ell_{2}$ и $c_{0}-$ обладающие свойством: в каждом бочечном ЛВП, имеющем $\ell$-абсолютный базис, все безусловные шаудеровские базисы $\ell$-абсолютны.

Ненормируемое ЛВП, в отличие от банахова пространства, может иметь безусловный базис, не являющийся $\ell$-абсолютным. Например, последовательность координатных ортов $\left(e_{n}\right)_{1}^{\infty}$ образует безусловный базис в $\mathscr{F}$-пространстве

$$
\ell^{p+}=\left\{\left(t_{n}\right)_{1}^{\infty}:\left(\sum_{n}\left|t_{n}\right|^{m_{r}}\right)^{\frac{1}{m_{r}}}=\left|\left(t_{n}\right)_{1}^{\infty}\right|_{r}<\infty, \quad m_{1}>m_{2}>\cdots>m_{r}>\cdots>p\right\},
$$

но не является $\ell$-абсолютным базисом ни при каком $\ell=(\ell,\|\cdot\|) \in \Lambda$. Действительно, в противном случае определяющая система норм $\left(|\cdot|_{r}\right)_{1}^{\infty}$ в $\ell^{p+}$ эквивалентна единственной норме $\left\|\left(t_{n}\right)_{1}^{\infty}\right\|=\left\|\left(t_{n}\left|e_{n}\right|_{r}\right)_{1}^{\infty}\right\| \quad\left(\left(t_{n}\right)_{1}^{\infty} \in \ell^{p+}, r=1,2, \ldots\right)$. Но $\ell^{p+}$ не нормируемо, поскольку не содержит ограниченной окрестности нуля. Мы покажем, что $\ell^{p+}$ вообще не имеет $\ell$-абсолютного базиса.

Пусть $X$ и $Y \subset X$ - банаховы пространства с единичными окрестностями нуля, соответственно, $U$ и $V$. Как известно [18]-[20], n-поперечником по Бернштейну множества $V$ (относительно $U$ ) называют число

$$
b_{n}(V, U)=\sup _{X_{n}} \inf \left\{\frac{\|x\|_{U}}{\|x\|_{V}}: x \in X_{n}, \quad x \neq 0\right\}, \quad n=1,2, \ldots,
$$

где sup берется по всем $n$-мерным подпространствам $X_{n}$ пространства $X$. Точные значения относительных $n$-поперечников для стандартных окрестностей нуля вложенных координатных пространств Орлича вычислены в [21]. В частности, если $U_{r}$ - единичная окрестность нуля в пространстве $\ell_{m_{r}}, r=1,2, \ldots$, то при любых $r_{1}<r_{2} \quad\left(=m_{r_{1}}>m_{r_{2}}>p\right)$ имеем

$$
b_{n}(V, U)=\frac{1}{n^{1 / m_{r_{2}}-1 / m_{r_{1}}}} \quad n=1,2, \ldots .
$$

Предположим далее, что $\ell=(\ell,\|\cdot\|) \in \Lambda$ и $\left(x_{n}\right)_{1}^{\infty}-\ell$-абсолютный базис в $\ell^{p+}$. Обозначим

$$
V_{s}=\left\{\left(t_{n}\right)_{1}^{\infty} \in \ell^{p+}:\left\|\left(t_{n}\left|x_{n}\right|_{s}\right)_{n=1}^{\infty}\right\|=\left|\left(t_{n}\right)_{1}^{\infty}\right|_{s}^{*} \leqslant 1\right\}, \quad s=1,2, \ldots
$$

Пользуясь тем, что определяющие системы окрестностей нуля $\left(U_{r}\right)_{1}^{\infty}$ и $\left(V_{r}\right)_{1}^{\infty}$ в $\ell^{p+}$ эквивалентны, выберем множества $U_{r_{1}}, V_{s_{1}}, U_{r_{2}} \neq U_{r_{3}}, V_{s_{2}}, U_{r_{4}}$ так, чтобы каждое следующее поглощалось предыдущим. Тогда в силу монотонности поперечников $b_{n}(V, U)$ и по $V$, и по $U$ справедлива оценка

$$
b_{n}\left(V_{s_{2}}, V_{s_{1}}\right) \leqslant C b_{n}\left(U_{r_{3}}, U_{r_{2}}\right)=\frac{C}{n^{1 / m_{r_{3}}-1 / m_{r_{2}}}}, \quad n=1,2, \ldots,
$$


с положительной постоянной $C$, не зависящей от $n$.

Покажем, что выполняется соотношение

$$
\lim _{k \rightarrow \infty} \frac{\left|x_{k}\right|_{s_{1}}}{\left|x_{k}\right|_{s_{2}}}=0
$$

Действительно, в противном случае при некотором $\delta>0$ для бесконечной последовательности индексов $\left(k_{j}\right)_{1}^{\infty} \subset \mathbb{N}$ будем иметь

$$
\frac{\left|x_{k_{j}}\right|_{s_{1}}}{\left|x_{k_{j}}\right|_{s_{2}}} \geqslant \delta, \quad j=1,2, \ldots
$$

Пусть $n$ фиксировано и $X_{n} \subset \ell^{p+}-$ подпространство, натянутое на первые $n$ элементов последовательности $\left(x_{k_{j}}\right)_{j=1}^{\infty}$. Из (3) и монотонности нормы $\|\cdot\|$ в пространстве $\ell$ для произвольного элемента $x=\sum_{j=1}^{n} u_{j} x_{k_{j}} \in X_{n}, x \neq 0$, выводим

$$
|x|_{s_{2}}^{*}=\left\|\sum_{j=1}^{n} u_{j}\left|x_{k_{j}}\right|_{s_{2}} e_{j}\right\|=\left\|\sum_{j=1}^{n} u_{j}\left|x_{k_{j}}\right| s_{1} \frac{\left|x_{k_{j}}\right|_{s_{2}}}{\left|x_{k_{j}}\right|_{s_{1}}} e_{j}\right\| \leqslant \frac{1}{\delta}\left\|\sum_{j=1}^{n} u_{j}\left|x_{k_{j}}\right|_{s_{1}} e_{j}\right\|=\frac{|x|_{s_{1}}^{*}}{\delta} .
$$

Отсюда следует выполнение оценки $b_{n}\left(V_{s_{2}}, V_{s_{1}}\right) \geqslant \delta$, которая противоречит (1). Этим доказано (2). Но тогда, как известно [22], каноническое отображение $\pi\left(V_{s_{2}}\right.$, $V_{s_{1}}$ ) банахова пространства $\left(\ell^{p+},|\cdot|_{s_{2}}^{*}\right)$ (пополнения $\ell^{p+}$ по норме $\left.|\cdot|_{s_{2}}^{*}\right)$ в пространство $\left(\ell^{p+},|\cdot|_{s_{1}}^{*}\right)$ компактно. Так как окрестности нуля $V_{s_{1}}$ и $U_{r_{4}}$ поглощаются, соответственно, окрестностями $U_{r_{1}}$ и $V_{s_{2}}$, тождественные отображения

$$
J\left(V_{s_{1}}, U_{r_{1}}\right):\left(\ell^{p+},|\cdot|_{s_{1}}^{*}\right) \rightarrow\left(\ell^{p+},|\cdot|_{r_{1}}\right) \quad \text { и } \quad J\left(U_{r_{4}}, V_{s_{2}}\right):\left(\ell^{p+},|\cdot|_{r_{4}}^{*}\right) \rightarrow\left(\ell^{p+},|\cdot|_{s_{2}}\right)
$$

непрерывны. Следовательно, каноническое отображение

$$
\pi\left(U_{r_{4}}, U_{r_{1}}\right)=J\left(V_{s_{1}}, U_{r_{1}}\right) \circ \pi\left(V_{s_{2}}, V_{s_{1}}\right) \circ J\left(U_{r_{4}}, V_{s_{2}}\right):\left(\ell^{p+},|\cdot|_{r_{4}}^{*}\right) \rightarrow\left(\ell^{p+},|\cdot|_{r_{1}}\right)
$$

компактно, как суперпозиция одного компактного и двух непрерывных отображений. Но это невозможно, поскольку $\left(\ell^{p+},\left.|\cdot|\right|_{r_{4}} ^{*}\right)=\ell_{m_{r_{4}}},\left(\ell^{p+},|\cdot|_{r_{1}}^{*}\right)=\ell_{m_{r_{1}}}$ и, как легко видеть, вложение $\ell_{r} \rightarrow \ell_{s}$ при $r<s$ не является компактным оператором. Таким образом, пространство $\ell^{p+}$ не имеет $\ell$-абсолютного базиса. Другими словами, доказана

Теорема 1. Существует $\mathscr{F}$-пространство с безусловным базисом, не изоморфное обобщенному пространству Кёте.

Заметим в заключение, что $\ell^{p+}$ содержит дополняемое подпространство, изоморфное пространству Кёте. Более того, каждое ядерное степенное пространство Кёте конечного типа изоморфно некоторому дополняемому подпространству пространства $\ell^{p+}[23]$.

\section{СПИСОК ЦИТИРОВАННОЙ ЛИТЕРАТУРЫ}

[1] М. М. Драгилев, Базисы в пространствах Кёте, РГУ, Ростов-на-Дону, 1983, 2003.

[2] A. Dynin, B. Mitiagin, "Criterion for nuclearity in terms of approximative dimension", Bull. Acad. Polon. Sci. Ser. Math., 8:8 (1960), 535-540. 
[3] G. Köthe, O. Toeplitz, "Lineare Räume mit unendlich vielen Koordinaten und Ringe unendlicher Matrizen", J. Reine Angew. Math., 171 (1934), 251-270.

[4] E. R. Lorch, "Bicontinuous linear transformation in certain vector spaces", Bull. Amer. Math. Soc., 45:2 (1939), 564-569.

[5] И. М. Гельфанд, "Замечание к статье Н. К. Бари «Биортогональные системы и базисы в гильбертовом пространстве»", Учен. зап. МГУ. Сер. матем., 4:148 (1951), 224-225.

[6] A. Pełczyński, "Projections in certain Banach spaces", Studia Math., 19 (1960), 209-228.

[7] J. Lindenstrauss, M. Zippin, "Banach spaces with a unique unconditional basis", J. Funct. Anal., 3 (1969), 115-125.

[8] A. Pełczyński, J. Singer, "On non-equivalent bases and conditional bases in Banach spaces", Studia Math., 25:1 (1964), 5-25.

[9] W. Wojtinski, "On bases in certain countably Hilbert spaces", Bull. Acad. Polon. Sci. Ser. Math., 14:12 (1966), 681-684.

[10] J. Lindenstrauss, A. Pełczyński, "Absolutely summing operators in $L_{p}$ spaces and their applications", Studia Math., 29 (1968), 275-326.

[11] В. П. Захарюта, "О квазиэквивалентности базисов в конечных центрах гильбертовых шкал", Докл. АН СССР, 180:4 (1968), 786-788.

[12] Б. С. Митягин, “Эквивалентность базисов в гильбертовых шкалах”, Studia Math., 37:2 (1971), 111-137.

[13] В.П. Кондаков, Некоторые вопросы изоморфизма и базисов локально выпуклых пространств. Дисс. ... к.ф.-м.н., РГУ, Ростов-на-Дону, 1972.

[14] N. J. Kalton, "On absolute bases", Math. An., 200 (1973), 209-225.

[15] M. Zippin, "On perfectly homogeneous bases in Banach spaces", Israel J. Math., 4 (1966), $265-272$.

[16] В.П. Кондаков, Вопросы геометрии ненормируемых пространств, РГУ, Ростов-наДону, 1983.

[17] J. Lindenstrauss, L. Tzafriri, Classical Banach Spaces I, II, Springer-Verlag, Berlin, Heidelberg, New York, 1977, 1979.

[18] В. М. Тихомиров, "Об $n$-мерных поперечниках некоторых функциональных классов", Докл. АН СССР, 130:4 (1960), 734-737.

[19] В. М. Тихомиров, "Поперечники множеств в функциональном пространстве и теория наилучших приближений", УМН, 15:3 (1960), 81-120.

[20] В. М. Тихомиров, Некоторые вопросы теории приближений, МГУ, М., 1976.

[21] П. А. Чалов, "Поперечники по Бернштейну множеств в координатных пространствах Орлича", Теор. функи., функи. анализ и их прилож., Вып. 35. Респ. межвед. науч. сборник, Вища школа, Харьков, 1981, 119-123.

[22] Б. С. Митягин, "Аппроксимативная размерность и базисы в ядерных пространствах", УMH, 16:4 (1961), 63-132.

[23] G. Metafune, V. B. Moscatelli, "On the space $\ell^{p+}=I_{q>p} \ell^{q}$ ", Math. Nachr., 147 (1990), $7-12$.

М. М. Драгилев

Поступила в редакцию

Ростовский государственный университет

04.06 .2004

\section{П. А. Чалов}

Ростовский государственный университет

E-mail: chalov@math.rsu.ru 